\title{
Pedunculopontine Nucleus Region Deep Brain Stimulation in Parkinson Disease: Surgical Techniques, Side Effects, and Postoperative Imaging
}

\author{
Clement Hamani ${ }^{a, b}$ Andres M. Lozano ${ }^{a}$ Paolo A.M. Mazzone ${ }^{c}$ Elena Moro ${ }^{d}$ \\ William Hutchison $^{\text {a }}$ Peter A. Silburn ${ }^{\text {e }}$ Ludvic Zrinzo $^{f}$ Mesbah Alam ${ }^{g}$ \\ Laurent Goetz $^{\text {h }}$ Erlick Pereira ${ }^{i}$ Anand Rughani ${ }^{a}$ Wesley Thevathasan ${ }^{j}$ Tipu Aziz ${ }^{i}$ \\ Bastiaan R. Bloem ${ }^{i}$ Peter Brown' Stephan Chabardes ${ }^{\text {h }}$ Terry Coynel Kelly Foote ${ }^{m}$ \\ Edgar Garcia-Rill ${ }^{\mathrm{n}}$ Etienne C. Hirsch ${ }^{\circ}$ Michael S. Okun ${ }^{\mathrm{p}}$ Joachim K. Krauss ${ }^{9}$
}

${ }^{a}$ Division of Neurosurgery, Toronto Western Hospital, University of Toronto, and ${ }^{\mathrm{b}}$ Research Imaging Centre, Centre for Addiction and Mental Health, Campbell Family Mental Health Research Institute, Toronto, Ont., Canada; ${ }^{\mathrm{C} O U}$ for Stereotactic and Functional Neurosurgery, ASLRMC - CTO Hospital, Roma, Italia; ${ }^{d}$ Department of Neurology-CHU,

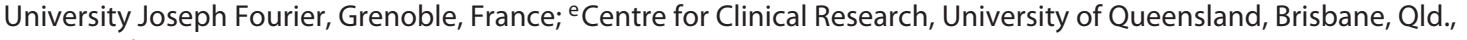
Australia; ${ }^{f}$ Unit of Functional Neurosurgery, UCL Institute of Neurology, London, UK; ${ }^{9}$ Department of Neurosurgery, Medical School Hannover, Hannover, Germany; ${ }^{\text {h }}$ Grenoble Institute of Neurosciences-INSERM U836 CEA-UJF-CHU, Department of Neurosurgery-CHU, University Joseph Fourier, Grenoble, France; 'Department of Neurosurgery, John Radcliffe Hospital, University of Oxford, Oxford, UK; ${ }^{j}$ Melbourne Brain Centre, Department of Medicine, University of Melbourne and Bionics Institute of Australia, Melbourne, Vic., Australia; ${ }^{2}$ Department of Neurology, Radboud University Medical Center, Nijmegen, The Netherlands; 'Department of Clinical Neurology, John Radcliffe Hospital, University of Oxford, Oxford, UK; ${ }^{m}$ Department of Neurosurgery, University of Florida, Gainesville, Fla., USA, ${ }^{n}$ Department of Anatomy and Neurobiology, University of Arkansas for Medical Sciences, Little Rock, Ark., USA; ${ }^{\circ}$ Institut du Cerveau et de la Moelle épinière, ICM, INSERM, CNRS, UMR 7225, Sorbonne Universités, UPMC Université, Paris, France; P University of Florida Center for Movement Disorders, Departments of Neurology and Neurosurgery, Gainesville, Fla., USA

\section{Key Words}

Brainstem · Deep brain stimulation · Imaging •

Pedunculopontine nucleus · Parkinson disease .

Side effects · Stereotactic technique

\begin{abstract}
The pedunculopontine nucleus (PPN) region has received considerable attention in clinical studies as a target for deep brain stimulation (DBS) in Parkinson disease. These studies have yielded variable results with an overall impression of improvement in falls and freezing in many but not all patients treated. We evaluated the available data on the surgi-
\end{abstract}

cal anatomy and terminology of the PPN region in a companion paper. Here we focus on issues concerning surgical technique, imaging, and early side effects of surgery. The aim of this paper was to gain more insight into the reasoning for choosing specific techniques and to discuss shortcomings of available studies. Our data demonstrate the wide range in almost all fields which were investigated. There are a number of important challenges to be resolved,

The authors constitute those members of the MDS Pedunculopontine Nucleus DBS Working Group in collaboration with the WSSFN who were charged with the domains 'surgical anatomy' and/or 'surgical technique'.

\section{KARGER}

E-Mail karger@karger.com

www.karger.com/sfn (c) 2016 S. Karger AG, Basel

1011-6125/16/0945-0307\$39.50/0 
such as identification of the optimal target, the choice of the surgical approach to optimize electrode placement, the impact on the outcome of specific surgical techniques, the reliability of intraoperative confirmation of the target, and methodological differences in postoperative validation of the electrode position. There is considerable variability both within and across groups, the overall experience with PPN DBS is still limited, and there is a lack of controlled trials. Despite these challenges, the procedure seems to provide benefit to selected patients and appears to be relatively safe. One important limitation in comparing studies from different centers and analyzing outcomes is the great variability in targeting and surgical techniques, as shown in our paper. The challenges we identified will be of relevance when designing future studies to better address several controversial issues. We hope that the data we accumulated may facilitate the development of surgical protocols for PPN DBS.

(c) 2016 S. Karger AG, Basel

The pedunculopontine nucleus (PPN) region has been used in clinical studies as a target for deep brain stimulation (DBS) in patients with Parkinson disease (PD) and gait disorders which were poorly responsive to medical treatment. As indicated in a companion paper, these studies have yielded variable results with an overall tendency for improvement in falls and freezing in many but not all treated patients (see companion paper on surgical anatomy). It remains unclear whether the variability in published data is related to patient selection, patient anatomy, differences in surgical technique or programming for chronic stimulation. It is likely that also multiple other factors may influence outcome.

We analyzed data on surgical anatomy and terminology of the PPN region in a companion paper. Here we focus on issues concerning surgical technique, imaging, and early side effects of surgery. The aim of this paper is to gain more insight into the reasoning for choosing specific techniques and to discuss shortcomings of available studies. Finally, this set of data is intended to form a foundation for developing a core protocol to be used in PPN DBS surgery.

\section{Methodological Approach}

The MDS Pedunculopontine Nucleus DBS Working Group in collaboration with the World Society for Stereotactic and Functional Neurosurgery (WSSFN) de- fined four domains to be addressed with regard to their relevance for PPN DBS surgery: preoperative selection of patients and available rating scales, clinical outcome and DBS programming, surgical anatomy, and surgical technique. In order to address the domain surgical technique, the executive committee formulated several questions during a consensus conference concerning also postoperative imaging and side effects which were distributed to the co-authors of the paper. Specific topics included unilateral versus bilateral implantation of DBS electrodes, combining PPN with other targets, stereotactic targeting, microelectrode recording, intraoperative test stimulation, type of DBS electrode, local field potentials, intraoperative and early postoperative complications, and postoperative imaging studies. A bibliometric analysis was performed in the PubMed database using the following key words: pedunculopontine nucleus; deep brain stimulation; anatomy; physiology; surgery.

Specific topics were assigned to groups of authors, and this work was accumulated and reviewed by the executive committee of the working group. Areas of disagreement were discussed and modified accordingly until a consensus could be reached. The literature was continuously updated during that process.

\section{Rationales for Bilateral versus Unilateral PPN DBS}

\section{Preclinical Data}

Semba and Fibiger [1] showed in rodents that the PPN input from the brainstem reticular formation, the lateral hypothalamus, the zona incerta, the midbrain central gray, the ventral tegmental area, the substantia nigra pars compacta, and the substantia nigra pars reticulata is primarily bilateral, but with an ipsilateral predominance. In contrast to those findings, other reports in rodents [2] and primates [3] have demonstrated that nucleus subthalamicus (STN) projections to the PPN were exclusively ipsilateral.

Kita and Kita [4] quantified the lateralization of projections from the PPN to the STN in rats. They observed that only $10 \%$ of projection neurons were cholinergic with a fivefold predominance of ipsilateral versus contralateral fibers. The predominance of ipsilateral to contralateral projections in the remaining noncholinergic projections was close to threefold.

In summary, projections from the basal ganglia and other structures to the PPN in rodents were predominantly unilateral. Those between PPN and the STN ap- 
peared to be bilateral, with a stronger ipsilateral representation. Although no data exist in humans, preclinical findings suggest that, even though unilateral surgery may influence both sides of the body, it may likely have a stronger impact on the contralateral side.

\section{Clinical Data}

Clinical insight into the safety and efficacy of unilateral versus bilateral PPN stimulation has been limited. Unilateral DBS has been carried out in two series with 6 [5] and 13 patients [6] treated in different centers. In contrast, bilateral DBS was conducted in various other trials. After an initial study with 2 individual patients [7], 2 series of 6 subjects were reported in which bilateral PPN DBS was delivered along with STN DBS $[8,9]$. Another series of 5 patients was reported with bilateral PPN DBS administered in tandem with DBS in the caudal zona incerta (cZI) [10]. A recent study which included a doubleblind randomized study protocol applied bilateral DBS to the anterior PPN $[11,12]$.

Imaging studies in PD patients suggest that unilateral PPN DBS increases cerebral blood flow bilaterally into the central thalamus and cerebellum [13]. Formal comparisons between unilateral and bilateral PPN DBS, however, tend to support bilateral DBS [14]. Khan et al. [14] showed that on-medication UPDRS motor scores improved by $5.7 \%$ with unilateral PPN DBS and by $18.4 \%$ with bilateral PPN DBS in a series of 5 PD patients. Similarly, improvement in UPDRS axial subscores (items 2730 ) after unilateral PPN DBS was $22 \%$ and after bilateral PPN DBS 36\%.

In an experimental study to measure the spatiotemporal characteristics of gait during PPN DBS, Thevathasan et al. [15] compared the effects of unilateral versus bilateral stimulation in a blinded fashion. The authors found that improvement in freezing after bilateral stimulation of the caudal PPN in the off-medication condition was approximately twice as high as that recorded after unilateral stimulation.

\section{Conclusions}

Both unilateral and bilateral PPN DBS were found to have an impact on gait and axial symptoms. Only 1 clinical trial and 1 study on gait compared the effects of bilateral versus unilateral PPN DBS directly. In both, bilateral stimulation proved to be superior, particularly for controlling freezing of gait. Nevertheless, these findings need to be corroborated by additional studies with a longer follow-up including a higher number of patients prior to specific recommendations.

Surgical Techniques, Side Effects, and Imaging in PPN DBS for PD

\section{Combination with Other Targets}

While few PD patients present with a predominant gait disorder or freezing refractory to medical treatment, the majority of patients in whom DBS is considered suffer also from other parkinsonian symptoms. Chronic stimulation of the PPN has been combined with stimulation of other targets, including the STN, the globus pallidus internus (GPi), and the cZI. There has been a different rationale for choosing each target with some evidence suggesting that target combinations may be superior to PPN DBS alone. Combined stimulation poses a series of challenges for the assessment of the effects of DBS in each target, as well as for unlocking the understanding of the complex relationship between medication and stimulation. A particular problem is the fact that, in general, most groups use low-frequency stimulation for the PPN and high-frequency stimulation in other targets. This regimen requires complex programming or the use of an additional pulse generator.

In their initial trial, Stefani et al. [8] implanted $6 \mathrm{PD}$ patients with bilateral PPN and STN electrodes. This combined approach was selected to tailor DBS according to the patients' symptoms - that is those typically controlled by STN DBS and those considered as being STN stimulation refractory [8]. Overall, the results showed a synergistic effect between targets. In a study from Grenoble, PPN DBS was added to STN DBS in 6 patients with advanced PD [16]. The addition of PPN DBS reduced the duration of freezing episodes under the off-drug condition. Moreover, it reduced falls related to freezing. Total UPDRS scores, however, did not significantly change.

In another report, in $3 \mathrm{PD}$ patients PPN DBS was combined with GPi DBS with the rationale to treat off dystonia as well as gait features [6]. In a more recent study, it was demonstrated that low-frequency PPN DBS combined with high-frequency GPi DBS in a patient with advanced $P D$ had a marked impact on gait ignition and freezing of gait. In contrast, isolated PPN or GPi DBS yielded only mild effects with the patient trial being blinded in each condition and by using a computerized gait analysis system [17].

Khan et al. $[10,18]$ observed that the effects of combined PPN and cZI DBS in 7 patients were superior to those of a single target. When both PPN and cZI electrodes were bilaterally activated, motor scores improved by $41.8 \%$. When receiving bilateral PPN and cZI DBS, UPDRS axial subscores improved by $58.3 \%$. 


\section{Conclusions}

Only a few trials have studied the effects of combining PPN DBS with stimulation of the STN, GPi or cZI. Furthermore, the small number of patients in each study limits the interpretation of the data.

\section{Stereotactic Targeting}

\section{Target Site within the PPN}

There is no consensus on the optimal target site within the PPN region. There remains uncertainty as to whether electrodes should be implanted in the rostral PPN (at the level of the inferior colliculus, IC) or caudal PPN (in a region about $4 \mathrm{~mm}$ below the IC). Given the murkiness of the available data and since the PPN is oriented along the long axis of the brainstem, a reasonable approach would be to have contacts in both rostral and caudal PPN regions.

It should be noted, however, that in animal studies of PPN stimulation-induced locomotion, the activation of the posterior pars compacta was efficacious while stimulation of the anterior PPN was not $[19,20]$. A recent study on an animal model of PD showed that stimulation of the anterior PPN induced freezing and worsened gait, but gait was improved by posterior PPN stimulation [21].

Fig. 1. Axial sections perpendicular to the long axis of the brainstem. Row I: level of the rostral PPN and mid-IC (IA and IB: 36 $\mathrm{mm}$ rostral to obex; IC and ID: $16 \mathrm{~mm}$ rostral to B-F plane). Row II: through the middle of the PPN (IIA and IIB: $33 \mathrm{~mm}$ rostral to obex; IIC and IID: $13 \mathrm{~mm}$ rostral to B-F plane). Row III: sections taken through the caudal PPN (IIIA and IIIB: $31 \mathrm{~mm}$ rostral to obex; IIIC and IIID: $11 \mathrm{~mm}$ rostral to B-F plane). Columns A and B: adapted from Paxinos and Huang [54]. Column A presents negative photographs of sections stained with cresyl violet and acetylcholinesterase: white matter appears dark, gray matter appears light. The line drawings presented in column B show the outline of fiber tracts and nuclei. The ventricular system is solid black, a number of structures relevant to the PPN have been assigned a color. Columns C and D: taken from Afshar et al. [55]. Column C presents photomicrographs of modified Mulligan stain sections: white matter tracts appear light, gray matter is dark. Column D: stereotactic drawings based upon probability data acquired from multiple hemibrainstems (see text, and note the superimposed stereotactic grid). Major structures are labeled, but the PPN is not amongst them. Surrounding structures have been assigned the same color scheme as in column B. Columns E and F: MRI images from a representative patient in the study acquired using the specifically modified proton density sequence described in the text.
Targeting of PPN across Different Groups

Below, we summarize the methods used by different investigators to target the PPN. Stereotactic imaging consisted of either MRI alone or combined with CT $[10,18]$.

Bristol, UK [7, 22]. T2-weighted MRI and proton density sequences were used. The PPN was defined on an axial plane acquired parallel to that formed by the upper border of the pons and the midbrain collicular point. In that section, the PPN was localized between the decussation of the superior cerebellar peduncle and the medial lemniscus. The nucleus was suggested to extend approximately $5 \mathrm{~mm}$ caudal to that point, running parallel to the fourth ventricle and to the cerebral aqueduct.

Toronto, Canada [23, 24]. T1- and T2-weighted MRI sequences were obtained. The target region was defined as being $4-5 \mathrm{~mm}$ below the caudal aspect of the IC, $2-3$ $\mathrm{mm}$ posterior to the limit between the base and tegmentum of the pons in the anteroposterior axis, and 3-4 $\mathrm{mm}$ medial to the lateral edge of the brainstem. The final coordinates were modified according to individual anatomical variations and by intraoperative findings. This target area represents the position of the lowest contact on the Medtronic 3387 DBS electrode. As the electrode has 4 contacts, the area of each of the other contacts, up to 10.5 $\mathrm{mm}$ above this chosen target, can be used for testing and chronic stimulation.
White matter appears hypointense and gray matter relatively hyperintense. Planning software was used to reconstruct axial images at a plane perpendicular to the midline of the fourth ventricular floor. Column F: interpretation of the structural arrangement of brainstem structures within the MR image assisted by the inherent image contrast as well as an understanding of the regional anatomy. The PPN can be directly localized within the gray matter lying lateral to the superior cerebellar peduncle and its decussation (green) and central tegmental tract (blue) and medial to the lemniscal systems (yellow) and is represented by a red circle. Note: the atlas photographs and drawings reproduced here were originally published in conventional anatomical representation (anterior surface of the brainstem towards the bottom of the page). These images were purposefully rotated through $180^{\circ}$ to make their orientation analogous to the axial radiological imaging convention that is more familiar to clinicians. CTT/ctg = Central tegmental tract LL/ll = lateral lemniscus; $\mathrm{ML}=$ medial lemniscus; $\mathrm{PPN}=$ pedunculopontine nucleus; $\mathrm{PPTgC}=\mathrm{PPN}$ pars compacta; $\mathrm{PPTgD}=$ $\mathrm{PPN}$ pars diffusa; SCP/scp = superior cerebellar peduncles; STT/ spth = spinothalamic tract. Illustrations in columns A and $\mathrm{B}$ reprinted from Paxinos and Huang [54]; illustrations in columns C and $\mathrm{D}$ reprinted from Afshar et al. [55]; adapted from Zrinzo et al. [29], with permission.

(For figure see next page.) 


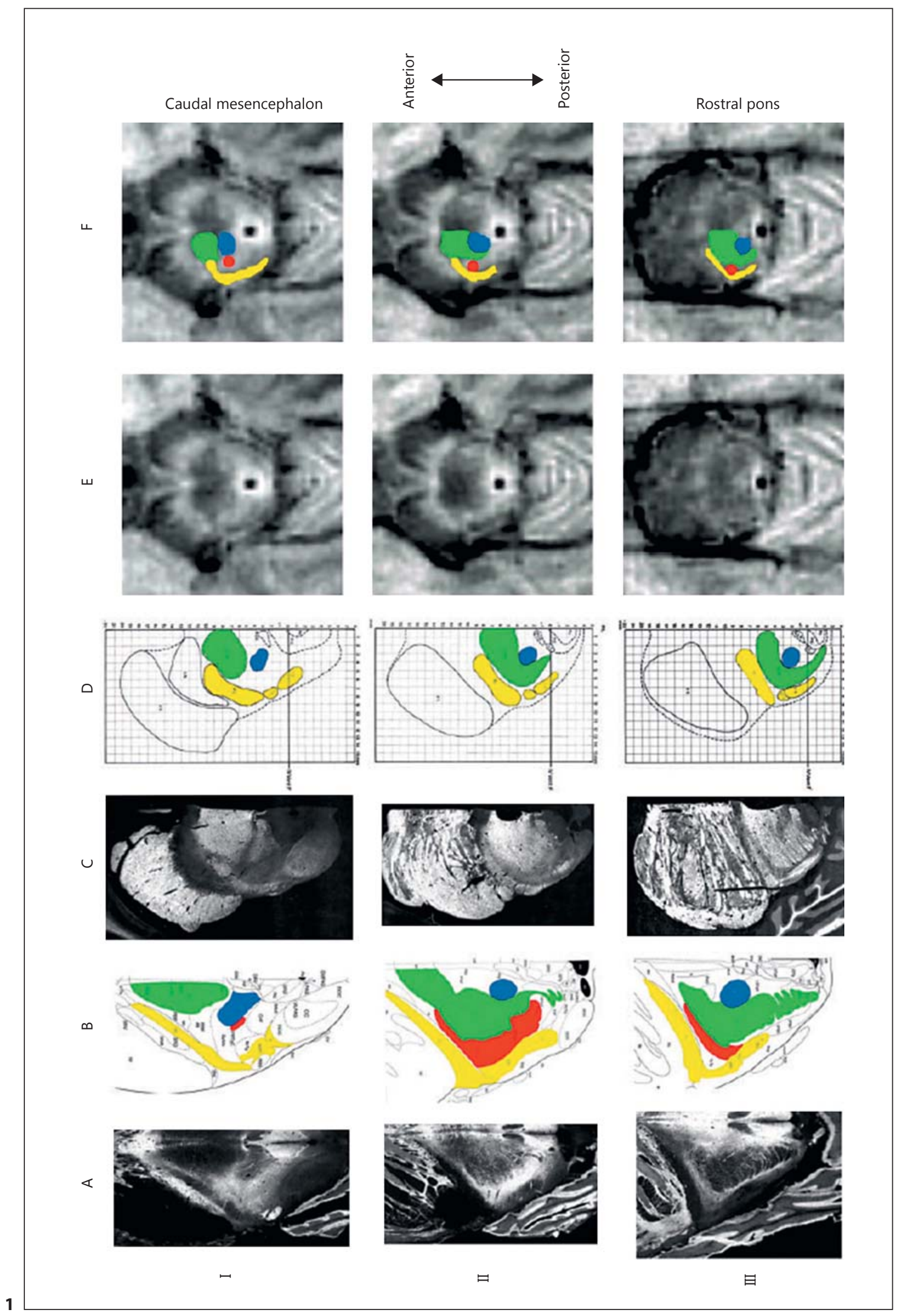




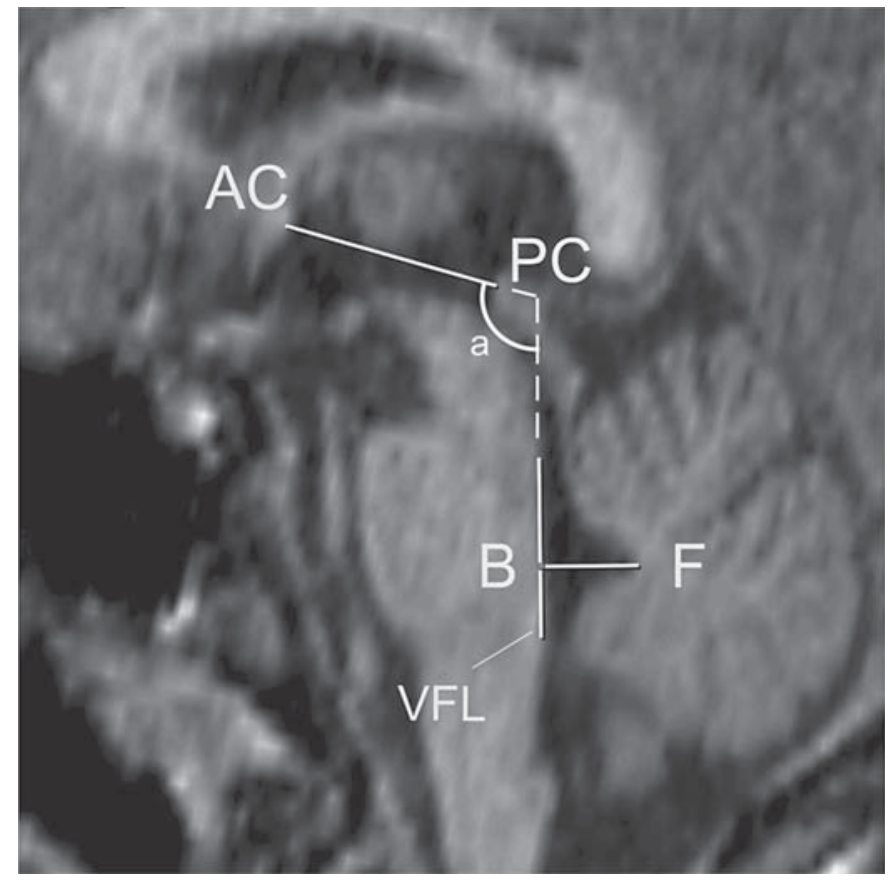

Fig. 2. Atlas-based landmarks of the fourth ventricle as defined in the stereotactic atlas of the human brainstem and cerebellum by Afshar et al. [55]. Variability in the angle of the mesencephalic flexure (a) may lead to increased variability in the spatial relationship of brainstem structures to the traditional AC-PC line. Brainstem structures may therefore enjoy a more constant relationship with fourth ventricular landmarks. A line is drawn tangentially to the floor of the fourth ventricle in the midline (VFL); a second line passes through the fastigium, perpendicular to the first. The intersection of these two lines (B) and the fastigial point (F) define two points in a new reference plane in a similar manner to that defined by the more traditional AC-PC points. Extensions of the VFL and the AC-PC line subtend an angle a: the mesencephalic angle. Adapted from Zrinzo et al. [29], with permission.

Oxford, UK [25, 26]. Patients underwent echo planar and diffusion-weighted MR data acquisition and diffusion tensor imaging. This allowed visualization of the superior cerebellar decussation and medial lemniscus. In addition, a stereotactic CT scan of the whole head at 1.0$\mathrm{mm}$ contiguous slices was acquired. Using planning software, the preoperatively acquired T2-weighted MRI scan was volumetrically fused to the stereotactic CT. The target chosen was the one delineated by diffusion tensor imaging. A trajectory was chosen along the long axis of the brainstem, from a level just below the red nucleus to the level below the IC, incorporating the seeded target site along the axis of implantation.
London, UK [27-29]. Zrinzo and colleagues used information derived from proton density MRI sequences to obtain an optimized visualization of the PPN and adjacent brainstem structures (fig. 1). The rostral pole of the PPN was identified at the mid-IC level, whereas the caudal pole was suggested to lie in the rostral pons. Altogether, the authors suggested that the PPN spans for a distance of $5 \mathrm{~mm}$. The authors characterized fourth ventricular landmarks, including the fastigial point, a line tangential to the floor of the fourth ventricle along the median sulcus (VFL), and a line perpendicular to the VFL passing through the fastigium. The intersection between these lines determined the base point. The axial plane to target the PPN was perpendicular to the VFL (fig. 2).

T1-weighted images were used to identify the landmarks and proton density images to provide contrast within the brainstem. On axial proton density images reformatted with respect to fourth ventricular landmarks, the rostral PPN was characterized as a region of intermediate signal intensity bounded laterally by the medial lemniscus, spinothalamic tract, and lateral lemniscus, anteromedially by the decussation of the superior cerebellar peduncle and posteromedially by the central tegmental tract. On more caudal proton density axial images $(5 \mathrm{~mm}$ inferior to the mid-IC level), the gray matter between the lemniscal system and the superior cerebellar peduncle was compressed to a narrow boomerang shape structure, and it was suggested that the caudal pole of the PPN occupies this slender gray matter region. Stereotactic images from 12 patients undergoing DBS of other brain targets were analyzed. Mean (and SD) coordinates for the visualized poles of the PPN in relation to the base pointfastigial (B-F) plane were derived. The rostral pole of the PPN was found to be $6(0.5) \mathrm{mm}$ lateral to the midline, and $4.2(0.8) \mathrm{mm}$ anterior and $19.3(1.4) \mathrm{mm}$ rostral to the base point. The caudal pole was $6.8(0.5) \mathrm{mm}$ lateral, 4.4 (0.5) $\mathrm{mm}$ anterior to $\mathrm{B}$, and 14.3 (1.4) $\mathrm{mm}$ rostral to the B-F plane.

Mean (and SD) coordinates of the MRI-localized PPN poles in relation to the anterior-posterior commissure (AC-PC) plane were as follows: rostral pole 6.0 (SD 0.5) $\mathrm{mm}$ lateral and $3.0(1.1) \mathrm{mm}$ posterior to the $\mathrm{PC}$, and 9.0 (1.1) mm caudal to the AC-PC plane. The caudal pole was $6.8(0.5) \mathrm{mm}$ lateral and $4.0(1.1) \mathrm{mm}$ posterior to the $\mathrm{PC}$, and 13.9 (1.2) mm caudal to the AC-PC plane. Coordinates of the PPN midpoint were $6.4(0.5) \mathrm{mm}$ lateral, 3.5 (1.0) $\mathrm{mm}$ posterior, and 11.4 (1.2) $\mathrm{mm}$ caudal to the PC. The authors indicated that there was considerable variation in the location of the PPN in relation to both third 
and fourth ventricular landmarks. They also pointed out that atlas-based coordinates would be useful in providing an estimate of PPN localization, but that further refinement by MRI would be needed.

Proof of principle of this method was also demonstrated by histologically verified accurate lead placement within the PPN bilaterally in a single cadaver study [28].

Rome, Italy [30, 31]. CT sections corresponding to an axial plane located $5 \mathrm{~mm}$ below the pontomesencephalic junction were obtained. The target was then located at a point within that section that was $7 \mathrm{~mm}$ in front of (lateral to) the wall of the pontine tegmentum. Investigators in Rome also used: (1) ventriculography; (2) angio-CT scans (axial planes), and (3) 3-dimensional (3D) reconstructions of brainstem atlases. As the trajectory was preferably extraventricular, the angles most commonly used ranged from 8 to $11^{\circ}$ in the coronal plane and $25^{\circ}$ in the sagittal plane, being as parallel as possible to the floor of the fourth ventricle.

Grenoble, France [32]. In Grenoble, investigators used ventriculography and MRI though there were changes in methodology which evolved over several years. Average coordinates of the tentative target were $1.5 \mathrm{~mm}$ posterior to the PC, $13 \mathrm{~mm}$ below the AC-PC line, and $6 \mathrm{~mm}$ lateral from the midline. The tentative trajectory was parallel to the floor of the fourth ventricle. In the first 6 patients, the PPN was targeted based on bi-orthogonal televentriculographic images, which allowed the localization of ventricular landmarks (AC, PC and aqueduct/floor of the fourth ventricle). These were fused with T1- and T2weighted 1.5-tesla MR scans. A central trajectory was chosen to be parallel to the floor of the fourth ventricle, passing through the $\mathrm{PC}$ with a 14 - to 20-degree mediolateral angle. The target depth was set at $13 \mathrm{~mm}$ below the $\mathrm{PC}$ and $6 \mathrm{~mm}$ from the midline. A second trajectory was defined $2 \mathrm{~mm}$ posterior to the central one. A third trajectory was occasionally performed $2 \mathrm{~mm}$ anterior or lateral to the central trajectory. In the next 5 patients, a target was chosen that was $2-3 \mathrm{~mm}$ posterior to the PC, $6 \mathrm{~mm}$ from the midline, at the level of the pontomesencephalic junction (PMJ), with an angle adapted to the brainstem orientation. Targeting was supported by using a brainstem-normalized coordinate system that accounted for the PMJ and its width as the craniocaudal and anteroposterior references, respectively.

San Francisco, USA [33]. This group targeted the PPN based on MRI at a point superolateral to the decussation of the superior cerebellar peduncles on T2-weighted fast spin echo images. Average coordinates were 6-7.5 mm lateral, $13-15 \mathrm{~mm}$ inferior, and $15-17 \mathrm{~mm}$ posterior to the midcommissural point. With respect to brainstembased coordinate systems, target coordinates were 6-7.5 $\mathrm{mm}$ lateral, 3-5 $\mathrm{mm}$ anterior to the base point, and 13-16 $\mathrm{mm}$ rostral to the B-F plane. When appropriate, the target was adjusted so that it would be $2-3 \mathrm{~mm}$ away from the lateral edge of the brainstem, at the level of the PMJ.

Brisbane, Australia [34]. After CT/MRI fusion, the PPN was targeted on MRI in stereotactic space with the concomitant use of reconstructions from brainstem atlases. The PPN was identified lateral to the superior cerebellar decussation and below the level of the IC. Trajectories were parallel to the axis of the brainstem and fourth ventricle, passing through the subthalamic region posterior to the red nucleus.

Hannover, Germany [17]. In Hannover, targeting was conducted based on T1- and T2-weighted MRI images fused with stereotactic CT. A combination of 3 different methods was used: (1) image-guided indirect determination of the PPN after the visualization of neighboring structures (decussation of the superior cerebellar peduncles and medial lemniscus); (2) preliminary definition of the midpoint of the PPN in relation to the AC-PC plane: 6-7 mm lateral, 3-4 $\mathrm{mm}$ posterior to the $\mathrm{PC}$, and 11-13 $\mathrm{mm}$ below the AC-PC plane; (3) preliminary definition of the rostral and the caudal poles of the PPN according to the method of Afshar (rostral pole: $6 \mathrm{~mm}$ lateral and 4 $\mathrm{mm}$ anterior to the base point, $16 \mathrm{~mm}$ rostral to the base B-F plane; caudal pole: $7 \mathrm{~mm}$ lateral and $4 \mathrm{~mm}$ anterior to the base point, $11 \mathrm{~mm}$ rostral to the B-F plane). The coordinates obtained by all 3 methods were then compared and adapted. The final target for the lowest electrode contact was the caudal pole of the PPN.

Paris, France [12]. The PPN was targeted using an MRI and a $3 \mathrm{D}$ histological atlas of the basal ganglia that were deformed to the preoperative T1-weighted MRI. A set of coordinates was obtained with each method, aiming for the lowest contact to be just outside the lower limit of the nucleus. The tentative target was defined by averaging these coordinates. Trajectories were selected taking into account the size of the brainstem and also avoiding the ventricles, caudate nuclei, and blood vessels.

\section{Conclusions}

Most authors advocate T2-weighted and proton density sequences for direct visualization of landmarks in the PPN region. The PPN target lies medial to the medial lemniscus, close to the lateral edge of the superior cerebellar commissure and lateral to the central tegmental tract. In the rostrocaudal axis, it straddles the PMJ with its rostral pole located at the level of the mid-IC. 
Most groups choose trajectories parallel to the floor of the fourth ventricle. According to some investigators, an appropriate dorsoventral landmark for placing the tip of the electrodes is a region $5 \mathrm{~mm}$ below the inferior edge of the IC (immediately inferior to the transition between midbrain and pons). The B-F system can be used for targeting but has only been validated by a few groups.

There is general agreement that indirect targeting based on the AC-PC plane only is problematic. Nevertheless, the reported average coordinates relative to the ACPC plane are the following: $6-7.5 \mathrm{~mm}$ lateral, $13-15 \mathrm{~mm}$ inferior, and $15-17 \mathrm{~mm}$ posterior to the AC-PC midpoint (or $1.5 \mathrm{~mm}$ posterior to the PC). A few authors also advocate that, in the medial lateral plane, the electrode tip should be placed in a region $2-4 \mathrm{~mm}$ medial to the lateral edge of the brainstem. Since the caudal extent of the PPN is unclear, some authors suggest placing the tip of the electrode $4 \mathrm{~mm}$ below the PMJ.

In conclusion, a variety of imaging protocols were used to approach the PPN. Although the target appears to be similar across studies, there is no consensus as to which protocol would be most appropriate.

\section{Microelectrode Recording}

\section{Electrophysiological Features of the PPN Region and}

Target Localization

Several groups reported the findings of microelectrode recording in the PPN region. The findings are summarized below.

Toronto, Canada [24]. In the study by the Toronto group, a total of 244 neurons were classified according to the width and polarity of their action potentials in 7 patients ( 2 with progressive supranuclear palsy). Three populations were described: (1) units that fired randomly at $17 \mathrm{~Hz}$ (most cells); (2) neurons suggested as being cholinergic based on the characteristics of their action potentials $(16 \%$ of the total number of cells firing at $9 \mathrm{~Hz})$, and (3) units with positive action potentials that fired regularly at a mean of $67 \mathrm{~Hz}$ (7\% of cells). Most neurons (57\%) fired randomly while $21 \%$ exhibited a bursty firing pattern. Above and below the PPN, the proportion of bursting neurons was smaller (11 and 19\%, respectively), while more neurons exhibited random firing (65 and 75\%, respectively). Overall, $36 \%$ of the neurons responded to at least one type of passive or voluntary limb movement. Changes in firing rate were mostly excitatory (about $80 \%)$.
Grenoble, France [35, 36]. Among 21 cells recorded, 14 (67\%) fired irregularly, and 7 (33\%) exhibited a burst-like pattern. The mean firing rate of all neurons was $28 \mathrm{~Hz}$. During locomotor tasks, $57 \%$ of cells modified their firing. Of interest, 2 patients were asked to mimic stepping movements in the operating room. In those individuals, 7 cells showed an increase in firing during mimicked gait.

San Francisco, USA [33]. The mean spontaneous discharge rate for neurons presumably within the PPN or dorsal to it was 23 and $35 \mathrm{~Hz}$, respectively. Bursting discharge was more prevalent in the PPN compared to more dorsal regions. Wide action potential neurons were seen in the region dorsal to the PPN, and in the dorsal part of the PPN itself, but not in caudal regions. In the region dorsal to the PPN, $44 \%$ of neurons had significant oscillatory activity, while this was only observed in $19 \%$ of cells within the PPN. Oscillation frequencies within the PPN tended to be higher than those recorded in dorsal regions. Responses to contralateral movements of the limbs were observed in $43 \%$ of units in the PPN region. Most units responded to passive movements with phasic increases in discharge.

Brisbane, Australia [37]. In a study from Brisbane, 686 cells were recorded from 11 patients (10 with PD and 1 with progressive supranuclear palsy). Neurons were divided into narrow (87\%) or wide-spike units (13\%). Mean firing rates in both populations were $12-13 \mathrm{~Hz}$. In narrow units, $16 \%$ of firing occurred in bursts, versus $46 \%$ in wide firing units. Wide units were more prominent in the caudal PPN and suggested to be cholinergic. Although around half of the cells recorded in this study were movement responsive, most units showed a decrease in firing (in contrast to other reports in the field). Interestingly, certain cells were inhibited during imaginary gait.

\section{Conclusions}

Most neurons in the PPN region seem to fire in a range between 12 and $30 \mathrm{~Hz}$ in patients with PD. There are however subpopulations that fire at lower $(9 \mathrm{~Hz}$; possible cholinergic cells) and higher rates $(60-70 \mathrm{~Hz})$. Most cells fire randomly. Around $20-30 \%$ of units fire in bursts, which are more commonly recorded in the presumed PPN than dorsal to it.

Around 35-45\% (perhaps more) of cells in the PPN region respond to passive or active movements. Some cells respond to movements that mimic gait patterns. The phenotype of cells recorded is unclear, and the exact nuclear region where the cells were recorded has also been difficult to pinpoint (PPN, cuneiform, or subcuneiform). 


\section{Intraoperative PPN Region Test Stimulation}

\section{Electrodes, Stimulation Parameters, Effects, and} Side Effects

According to published data, most groups do not use microstimulation to assess thresholds for possible side effects induced by electrical stimulation.

Based on the Grenoble experience in awake patients [36], stimulation induced no effect on akinesia, rigidity or tremor. Furthermore, no effects were observed during a task in which patients were asked to pedal while in the supine position. However, microstimulation was used to delineate neighboring structures. Stimulation was applied every $3 \mathrm{~mm}$ from -5 to $-15 \mathrm{~mm}$ below the AC-PC plane. Stimulation parameters were: frequencies of 25 and $130 \mathrm{~Hz}$, a pulse width of $0.06 \mathrm{~ms}$, and current intensities of 0.1 up to $4 \mathrm{~mA}$. Stimulation induced the following effects depending on frequency: mono- and binocular movements (such as eye deviation or trembling vision) at both 25 and $130 \mathrm{~Hz}$, mostly present when stimulation was delivered at the rostral level of the IC; myoclonic movements/muscular vibratory-like sensations at $25 \mathrm{~Hz}$; paresthesias, mainly at $130 \mathrm{~Hz}$, when stimulation was delivered laterally, around 6-7 $\mathrm{mm}$ from the midline.

Sites in which monocular deviation and paresthesias were recorded at $25 \mathrm{~Hz}$ at a low amplitude were avoided for lead implantation. Best clinical outcomes were obtained when $130-\mathrm{Hz}$ microstimulation induced only transient paresthesias on the contralateral face and upper limb, and when $25 \mathrm{~Hz}$ induced myoclonic movements/ muscular vibration-like sensations at the PMJ level. Some authors have suggested that this could be a possible target signature.

The Brisbane group reported stimulation-induced sleep when current was delivered at $100-130 \mathrm{~Hz}$ to the rostral PPN [38]. Other side effects included disturbances with ocular pursuit and saccades, with reports of shimmering in the visual fields.

\section{Conclusions}

Reported stimulation-induced effects in the operating room include oscillopsias, paresthesias, and myoclonic movements.

\section{What Type of DBS Electrode to Implant}

Both the Medtronic 3387 and the Medtronic 3389 DBS electrodes have been used (Medtronic Inc., Minneapolis, Minn., USA). The advantage of the 3387 is that, with a wider span of the contacts, a broader anatomical coverage is possible. Since the PPN is partially degenerated in PD (and more so in progressive supranuclear palsy [39-41]), one may advocate that smaller-spaced electrodes might be preferable. Given the length of the PPN region and the uncertainty about the optimal stimulation site, the Oxford group favors the 3387 electrode. Since the Brisbane group concentrated on the more caudal region of the PPN, the 3389 model was used.

\section{Conclusions}

To date, there is no data suggesting that one electrode is better than the other for stimulating the PPN region.

\section{Local Field Potentials}

Several studies have explored local field potentials in the PPN region, intraoperatively from microelectrodes $[24,33]$ or postoperatively from externalized DBS electrodes [38, 42]. Overall results have been heterogeneous with patients showing peaks in the theta, alpha or beta frequency bands $[24,33,38,42]$. One study suggested that this variability could be related to electrode location, with alpha and beta activity being more marked in the caudal and rostral PPN, respectively [38]. The San Francisco group demonstrated that, as the electrode was advanced, the percentage of power in the low beta range $(13-21 \mathrm{~Hz})$ increased from 35.2 to $40.8 \%$ at rest, and from 11.1 to $29.8 \%$ during movement $[24,33]$. In a few studies, local field potentials were shown to respond to limb movement $[33,42]$ and to correlate with gait (e.g. alpha band) [38]. In a study from Oxford, only the caudal alpha activity correlated with gait and freezing of gait whilst rostral beta activity did not [38]. Changes in electrophysiological activity were observed after L-dopa administration [43] and related to movement initiation [42]. Tattersall et al. [37] have recently demonstrated that alpha power was significantly higher relative to beta power in the caudal PPN. These authors have also found an increase in both alpha and beta power (alpha/beta power ratio) during limb movements. Relatively less attention has been paid to gamma band activity [44].

Additionally, somatosensory evoked potentials were recorded from implanted PPN electrodes [30, 45-47]. Yeh et al. [48] recorded somatosensory evoked potentials after median nerve stimulation. These were triphasic or biphasic potentials with latencies to the largest negative peak in the order of 16.8 and $18.7 \mathrm{~ms}$. There was no difference in somatosensory evoked potential amplitude 

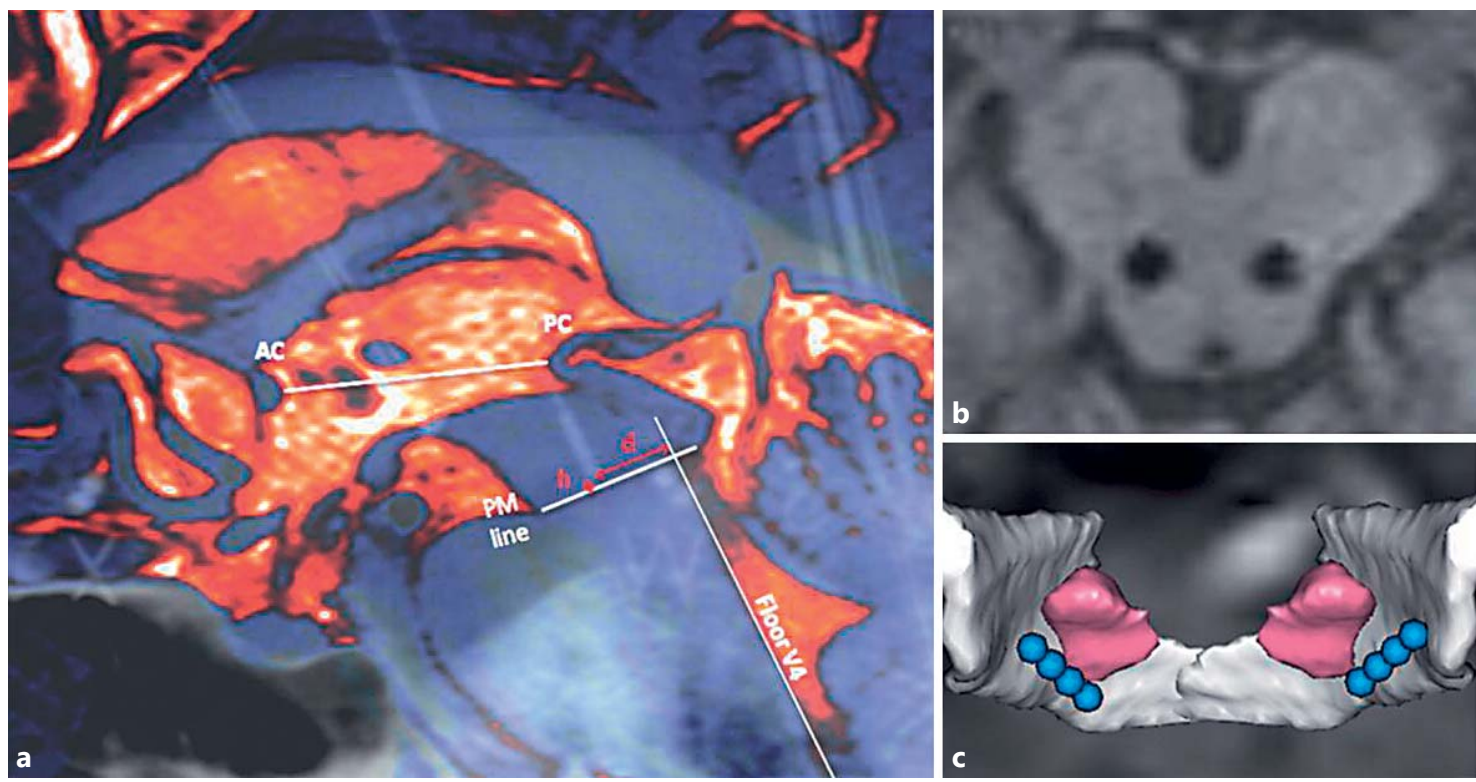

Fig. 3. Location of DBS electrodes in the PPN region in a patient from the Grenoble group. a Sagittal fusion imaging of the final intraoperative teleradiography with the preoperative MRI, showing how the coordinates of the tip of the distal contacts of the electrodes were measured. $\mathrm{h}=$ Distance (in millimeters) to the pontomesencephalic (PM) line, defined as the line connecting in the anterior-posterior direction the $\mathrm{PMJ}$ to the caudal end of the quadrigeminal plate, measured on the midline; $\mathrm{AC}=$ anterior commissure; $\mathrm{PC}=$ posterior commissure; $\mathrm{d}=$ orthogonal distance in millimeters to the line prolonging the fourth ventricle line; V4 = fourth ven-

and latency between on- and off-medication states. Highfrequency oscillations could be identified after the contralateral median nerve had been stimulated.

As part of the reticular activating system, the PPN may also respond directly to sensory inputs [20]. In humans, the midlatency auditory evoked $\mathrm{P} 50$ potential recorded at the vertex has been proposed to be generated by the PPN [49], but such studies have not been carried out in PD patients [50].

\section{Conclusions}

Frequency bands in the alpha, beta, and theta ranges and movement-related potentials were all recorded from the PPN region. In the off-medication condition, beta desynchronization occurred during movement in a small study. In the on-medication condition, oscillations in the 7- to $11-\mathrm{Hz}$ range were recorded. Beta-related synchronization occurred during premovement phases, and thetarelated desynchronization occurred with movement.

It may be important to develop a standard set of resting and movement-related intraoperative local field po- tricle. $\mathbf{b} 3 \mathrm{D}$ T1-weighted MRI in the axial plane parallel to the bicommissural plane, at the level of contacts 1 and 5 of the electrodes. Those contacts delivered cathodic current, and the contact depth of the right and left electrodes was symmetrical. c Atlas adaptation onto the patient's MRI. Superior posterior view of the 3D image with the PPN in pink, the medial lemniscus in white and the 4 electrode contacts in blue. Note that in this patient the electrode is located posterior to the PPN. Adapted from Ferraye et al. [16], with permission.

tentials that might help to optimize PPN targeting. One problem is that the alpha band activity correlating with gait may not be evident when patients are lying supine.

\section{Intraoperative and Early Postoperative Complications}

\section{Adverse Effects and Management}

Until recently, there have been no reports of serious adverse events either intraoperatively or in the early postoperative period after PPN DBS in more than 100 published patients. The report from the Paris group, however, reported 2 severe complications [11, 12]. Symptomatic bleeding occurred in 1 patient, who developed a midbrain hematoma, followed by impairment of consciousness. After recovery, he was discharged, wheel-chair bound, anarthric and had to be fed via gastrostomy. Another patient had an infection that required removal of the DBS system 1 month after surgery. He recovered without sequelae but decided not to be re-implanted. 


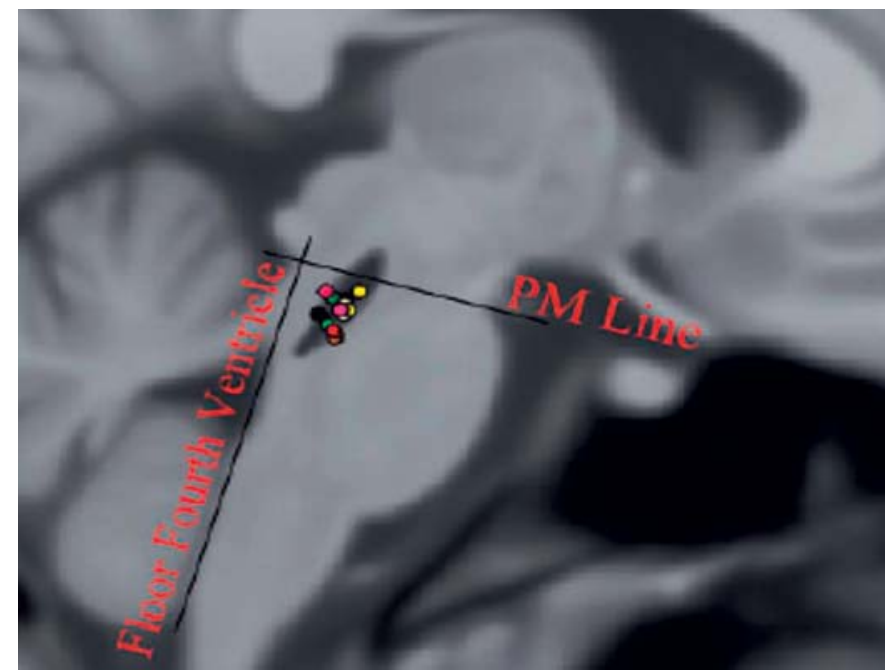

Fig. 4. Location of active stimulation sites within the PPN region mapped on a sagittal T1-weighted MR scan. Adapted from Thevathasan et al. [15], with permission.

Overall, available data suggest that PPN DBS surgery has a safety profile that is similar to other targets which are being used in PD; however, it should be noted that these findings may be underestimated due to a reporting bias.

Stimulation-induced adverse events in the early postoperative period included oscillopsia, paresthesias, burning sensations, myoclonus, sleep induction, and incontinence $[32,38,51]$. These phenomena were time locked with stimulation, they were frequency dependent, and were reversible when stimulation was reduced or ceased.

\section{Conclusions}

Available evidence suggests that surgery for implantation of electrodes in the PPN is relatively safe and, in general, well tolerated.

\section{Postoperative Imaging}

Examination of postoperative figures from published studies reveals that in most instances electrodes were implanted in apparently the same anatomical region although many studies do not provide much detail. There is both variation in the anteroposterior axis and in the depth of lead placement relative to the PMJ. When assessing coordinates and the exact numbers, it is difficult to reach uniform conclusions. Some studies reported the lo- cation of the tip of the electrodes, while others described the position of contacts 0 or 2. Further, studies have used different landmarks when assessing their data, including the AC-PC or the B-F system. Another issue is that, even when electrodes were placed in apparently the same brainstem region, patients were stimulated through different contacts (some in the caudal PPN, others in the rostral PPN) [6-8, 13, 17, 22, 23, 25, 28, 30, 52, 53].

Some MR sequences produce large lead artifacts and only poor internal tissue contrast within the brainstem. This may limit the accurate determination of the localization of the lead (fig. 3) [16]. Nevertheless, MRI is currently the most useful method to localize and to confirm electrode placement in the PPN region as well as to document its variability (fig. 4) [36, 38].

\section{Conclusions}

A wide variety of techniques were employed to document lead location following PPN DBS. CT imaging cannot account for individual anatomical variability and requires coregistration with atlases or MRimages. Although most authors used MRI, sequences were often not reported in detail. Poor tissue contrast within the region of interest appears to be a general problem, and this adds to the difficulty in identifying internal anatomical landmarks. Stereotactic MRI using proton density sequences seems to be of value for documenting lead placement in the PPN region postoperatively.

\section{Challenges and Future Prospects}

PPN DBS is a relatively novel intervention in PD. Our data here demonstrate the wide ranges in almost all fields which were investigated. There are a number of important challenges to be resolved such as identification of the optimal target, the choice of the surgical approach to optimize electrode placement, impact on outcome of specific surgical techniques, reliability of intraoperative confirmation of the target, and methodological differences in postoperative validation of the electrode position. There is considerable variability both within and across groups, the overall experience with PPN DBS is still limited, and there is a lack of controlled trials. Despite these challenges, the procedure seems to provide benefit to selected patients and appears to be relatively safe.

There is uncertainty of the future role of PPN DBS in the armamentarium of surgery for patients with PD. There is some risk that the benefits of this procedure 
would not be fully appreciated, since there has also been doubt about its overall efficacy and about its clear-cut benefits. Certainly, more studies are needed providing more solid data on the advantages and limits of chronic stimulation. One important limitation in comparing studies from different centers and analyzing outcome is the great variability in targeting and surgical techniques, as shown in our paper.

By systematically reviewing the published data, we identified several challenges which will be of relevance when designing future studies to better address controversial issues. We also hope that the data shown here may facilitate the development of surgical protocols for PPN DBS.

\section{Disclosure Statement}

The MDS Pedunculopontine Nucleus DBS Working Group in collaboration with the WSSFN was supported by an unrestricted grant from Medtronic.

\section{References}

1 Semba K, Fibiger HC: Afferent connections of the laterodorsal and the pedunculopontine tegmental nuclei in the rat: a retro- and antero-grade transport and immunohistochemical study. J Comp Neurol 1992;323:387-410.

2 Hammond C, Rouzaire-Dubois B, Feger J, Jackson A, Crossman AR: Anatomical and electrophysiological studies on the reciprocal projections between the subthalamic nucleus and nucleus tegmenti pedunculopontinus in the rat. Neuroscience 1983;9:41-52.

-3 Nauta HJ, Cole M: Efferent projections of the subthalamic nucleus: an autoradiographic study in monkey and cat. J Comp Neurol 1978;180:1-16.

4 Kita T, Kita H: Cholinergic and non-cholinergic mesopontine tegmental neurons projecting to the subthalamic nucleus in the rat. Eur J Neurosci 2011;33:433-443.

5 Moro E, Hamani C, Poon YY, Al-Khairallah T, Dostrovsky JO, Hutchison WD, Lozano AM: Unilateral pedunculopontine stimulation improves falls in Parkinson's disease. Brain 2010;133:215-224.

6 Mazzone P, Sposato S, Insola A, Scarnati E: The deep brain stimulation of the pedunculopontine tegmental nucleus: towards a new stereotactic neurosurgery. J Neural Transm 2011;118:1431-1451.

7 Plaha P, Gill SS: Bilateral deep brain stimulation of the pedunculopontine nucleus for Parkinson's disease. Neuroreport 2005;16:18831887.

8 Stefani A, Lozano AM, Peppe A, Stanzione P, Galati S, Tropepi D, Pierantozzi M, Brusa L, Scarnati E, Mazzone P: Bilateral deep brain stimulation of the pedunculopontine and subthalamic nuclei in severe Parkinson's disease. Brain 2007;130:1596-1607.

$\checkmark 9$ Ferraye MU, Debu B, Fraix V, Goetz L, Ardouin C, Yelnik J, Henry-Lagrange C, Seigneuret E, Piallat B, Krack P, Le Bas JF, Benabid AL, Chabardes S, Pollak P: Effects of pedunculopontine nucleus area stimulation on gait disorders in Parkinson's disease. Brain 2010;133:205-214.
10 Khan S, Javed S, Mooney L, White P, Plaha P, Whone A, Gill SS: Clinical outcomes from bilateral versus unilateral stimulation of the pedunculopontine nucleus with and without concomitant caudal zona incerta region stimulation in Parkinson's disease. Br J Neurosurg 2012;26:722-725.

11 Welter ML, Demain A, Ewenczyk C, Czernecki V, Lau B, El Helou A, Belaid H, Yelnik J, François C, Bardinet E, Karachi C, Grabli D: PPNa-DBS for gait and balance disorders in Parkinson's disease: a double-blind, randomised study. J Neurol 2015;262:15151525.

12 Lau B, Welter ML, Belaid H, Fernandez Vidal S, Bardinet E, Grabli D, Karachi C: The integrative role of the pedunculopontine nucleus in human gait. Brain 2015;138:1284-1296.

13 Ballanger B, Lozano AM, Moro E, van Eimeren T, Hamani C, Chen R, Cilia R, Houle S, Poon YY, Lang AE, Strafella AP: Cerebral blood flow changes induced by pedunculopontine nucleus stimulation in patients with advanced Parkinson's disease: a $\left[{ }^{15} \mathrm{O}\right] \mathrm{H}_{2} \mathrm{O}$ PET study. Hum Brain Mapp 2009;30:39013909.

14 Khan S, Javed S, Mooney L, White P, Plaha P, Whone A, Gill SS: Clinical outcomes from bilateral versus unilateral stimulation of the pedunculopontine nucleus with and without concomitant caudal zona incerta region stimulation in Parkinson's disease. Br J Neurosurg 2012;26:722-725.

15 Thevathasan W, Cole MH, Graepel CL, Hyam JA, Jenkinson N, Brittain JS, Coyne TJ, Silburn PA, Aziz TZ, Kerr G, Brown P: A spatiotemporal analysis of gait freezing and the impact of pedunculopontine nucleus stimulation. Brain 2012;135:1446-1454.

16 Ferraye MU, Debu B, Fraix V, Goetz L, Ardouin C, Yelnik J, Henry-Lagrange C, Seigneuret E, Piallat B, Krack P, Le Bas JF, Benabid AL, Chabardes S, Pollak P: Effects of pedunculopontine nucleus area stimulation on gait disorders in Parkinson's disease. Brain 2010;133:205-214.
17 Schrader C, Seehaus F, Capelle HH, Windhagen $\mathrm{A}$, Windhagen $\mathrm{H}$, Krauss JK: Effects of pedunculopontine area and pallidal DBS on gait ignition in Parkinson's disease. Brain Stimul 2013;6:856-859.

18 Khan S, Mooney L, Plaha P, Javed S, White P, Whone AL, Gill SS: Outcomes from stimulation of the caudal zona incerta and pedunculopontine nucleus in patients with Parkinson's disease. Br J Neurosurg 2011;25:273280.

19 Garcia-Rill E: The pedunculopontine nucleus. Prog Neurobiol 1991;36:363-389.

20 Reese NB, Garcia-Rill E, Skinner RD: The pedunculopontine nucleus - auditory input, arousal and pathophysiology. Prog Neurobiol 1995;47:105-133.

21 Gut NK, Winn P: Deep brain stimulation of different pedunculopontine targets in a novel rodent model of parkinsonism. J Neurosci 2015;35:4792-4803.

22 Khan S, Javed S, Park N, Gill SS, Patel NK: A magnetic resonance imaging-directed method for transventricular targeting of midline structures for deep brain stimulation using implantable guide tubes. Neurosurgery 2010; 66:234-237; discussion 237.

23 Moro E, Hamani C, Poon YY, Al-Khairallah T, Dostrovsky JO, Hutchison WD, Lozano AM: Unilateral pedunculopontine stimulation improves falls in Parkinson's disease. Brain 2010;133:215-224.

24 Weinberger M, Hamani C, Hutchison WD, Moro E, Lozano AM, Dostrovsky JO: Pedunculopontine nucleus microelectrode recordings in movement disorder patients. Exp Brain Res 2008;188:165-174.

-25 Pereira EA, Muthusamy KA, De Pennington N, Joint CA, Aziz TZ: Deep brain stimulation of the pedunculopontine nucleus in Parkinson's disease. Preliminary experience at $\mathrm{Ox}$ ford. Br J Neurosurg 2008;22(suppl 1):S41S44.

26 Pereira EA, Nandi D, Jenkinson N, Stein JF, Green AL, Aziz TZ: Pedunculopontine stimulation from primate to patient. J Neural Transm 2011;118:1453-1460. 
27 Zrinzo L, Zrinzo LV: Surgical anatomy of the pedunculopontine and peripeduncular nuclei. Br J Neurosurg 2008;22(suppl 1):S19S24.

28 Zrinzo L, Zrinzo LV, Massey LA, Thornton J, Parkes HG, White M, Yousry TA, Strand C, Revesz T, Limousin P, Hariz MI, Holton JL: Targeting of the pedunculopontine nucleus by an MRI-guided approach: a cadaver study. J Neural Transm 2011;118:1487-1495.

-29 Zrinzo L, Zrinzo LV, Tisch S, Limousin PD, Yousry TA, Afshar F, Hariz MI: Stereotactic localization of the human pedunculopontine nucleus: atlas-based coordinates and validation of a magnetic resonance imaging protocol for direct localization. Brain 2008;131: 1588-1598.

-30 Mazzone P, Insola A, Sposato S, Scarnati E: The deep brain stimulation of the pedunculopontine tegmental nucleus. Neuromodulation 2009;12:191-204.

- 31 Mazzone P, Sposato S, Insola A, Scarnati E: The clinical effects of deep brain stimulation of the pedunculopontine tegmental nucleus in movement disorders may not be related to the anatomical target, leads location, and setup of electrical stimulation. Neurosurgery 2013;73:894-906; discussion 905-906.

- 32 Ferraye MU, Gerardin P, Debu B, Chabardes S, Fraix V, Seigneuret E, LeBas JF, Benabid AL, Tilikete C, Pollak P: Pedunculopontine nucleus stimulation induces monocular oscillopsia. J Neurol Neurosurg Psychiatry 2009; 80:228-231.

- 33 Shimamoto SA, Larson PS, Ostrem JL, Glass GA, Turner RS, Starr PA: Physiological identification of the human pedunculopontine nucleus. J Neurol Neurosurg Psychiatry 2010; 81:80-86.

34 Wilcox RA, Cole MH, Wong D, Coyne T, Silburn P, Kerr G: Pedunculopontine nucleus deep brain stimulation produces sustained improvement in primary progressive freezing of gait. J Neurol Neurosurg Psychiatry 2011; 82:1256-1259.

- 35 Piallat B, Chabardes S, Torres N, Fraix V, Goetz L, Seigneuret E, Bardinet E, Ferraye M, Debu B, Krack P, Yelnik J, Pollak P, Benabid AL: Gait is associated with an increase in ton- ic firing of the sub-cuneiform nucleus neurons. Neuroscience 2009;158:1201-1205.

36 Goetz L, Maineri C, Piallat B, David O, Ferraye $M$, Torres N, Debu B, Seigneuret E, Fraix V, Lebas JF, Pollak P, Benabid AL, Chabardès $S$ : A normalized per-operatory microstimulation mapping of the rostral brainstem as a tool to delineate PPNa target. ESSFN Congr, Athens, 2010.

37 Tattersall TL, Stratton PG, Coyne TJ, Cook R, Silberstein P, Silburn PA, Windels F, Sah P: Imagined gait modulates neuronal network dynamics in the human pedunculopontine nucleus. Nat Neurosci 2014;17:449-454.

38 Thevathasan W, Pogosyan A, Hyam JA, Jenkinson N, Foltynie T, Limousin P, Bogdanovic M, Zrinzo L, Green AL, Aziz TZ, Brown P: Alpha oscillations in the pedunculopontine nucleus correlate with gait performance in parkinsonism. Brain 2012;135:148-160.

39 Hirsch EC, Graybiel AM, Duyckaerts C, Javoy-Agid F: Neuronal loss in the pedunculopontine tegmental nucleus in Parkinson disease and in progressive supranuclear palsy. Proc Natl Acad Sci USA 1987;84:5976-5980.

40 Jellinger K: The pedunculopontine nucleus in Parkinson's disease, progressive supranuclear palsy and Alzheimer's disease. J Neurol Neurosurg Psychiatry 1988;51:540-543.

41 Zweig RM, Whitehouse PJ, Casanova MF, Walker LC, Jankel WR, Price DL: Loss of pedunculopontine neurons in progressive supranuclear palsy. Ann Neurol 1987;22:18-25.

42 Tsang EW, Hamani C, Moro E, Mazzella F, Poon YY, Lozano AM, Chen R: Involvement of the human pedunculopontine nucleus region in voluntary movements. Neurology 2010;75:950-959.

43 Androulidakis AG, Mazzone P, Litvak V, Penny W, Dileone M, Gaynor LM, Tisch S, Di Lazzaro V, Brown P: Oscillatory activity in the pedunculopontine area of patients with Parkinson's disease. Exp Neurol 2008;211:59-66.

44 Garcia-Rill E, Kezunovic N, Hyde J, Simon C, Beck P, Urbano FJ: Coherence and frequency in the reticular activating system (RAS). Sleep Med Rev 2013;17:227-238.

$\checkmark 45$ Valeriani M, Truini A, Le Pera D, Insola A, Galeotti F, Petrachi C, Mazzone P, Cruccu G:
Laser evoked potential recording from intracerebral deep electrodes. Clin Neurophysiol 2009;120:790-795.

46 Insola A, Valeriani M, Mazzone P: Targeting the pedunculopontine nucleus: a new neurophysiological method based on somatosensory evoked potentials to calculate the distance of the deep brain stimulation lead from the obex. Neurosurgery 2012;71:96103.

47 Insola A, Padua L, Mazzone P, Scarnati E, Valeriani M: Low- and high-frequency somatosensory evoked potentials recorded from the human pedunculopontine nucleus. Clin Neurophysiol 2014;125:1859-1869.

48 Yeh IJ, Tsang EW, Hamani C, Moro E, Mazzella F, Poon YY, Lozano AM, Chen R: Somatosensory evoked potentials recorded from the human pedunculopontine nucleus region. Mov Disord 2010;25:2076-2083.

49 Erwin RJ, Buchwald JS: Midlatency auditory evoked responses in the human and the cat model. Electroencephalogr Clin Neurophysiol Suppl 1987;40:461-467.

50 Garcia-Rill E, Hyde J, Kezunovic N, Urbano FJ, Petersen E: The physiology of the pedunculopontine nucleus: implications for deep brain stimulation. J Neural Transm 2015;122: 225-235.

51 Arnulf I, Ferraye M, Fraix V, Benabid AL, Chabardes S, Goetz L, Pollak P, Debu B: Sleep induced by stimulation in the human pedunculopontine nucleus area. Ann Neurol 2010; 67:546-549.

52 Thevathasan W, Coyne TJ, Hyam JA, Kerr G, Jenkinson N, Aziz TZ, Silburn PA: Pedunculopontine nucleus stimulation improves gait freezing in Parkinson disease. Neurosurgery 2011;69:1248-1253; discussion 1254.

53 Ostrem JL, Christine CW, Glass GA, Schrock LE, Starr PA: Pedunculopontine nucleus deep brain stimulation in a patient with primary progressive freezing gait disorder. Stereotact Funct Neurosurg 2010;88:51-55.

54 Paxinos G, Huang XF: Atlas of the Human Brainstem. San Diego, Academic Press, 1995.

55 Afshar F, Watkins ES, Yap JC: Stereotaxic Atlas of the Human Brainstem and Cerebellar Nuclei. New York, Raven Press, 1978.
Surgical Techniques, Side Effects, and Imaging in PPN DBS for PD
Stereotact Funct Neurosurg 2016;94:307-319 DOI: $10.1159 / 000449011$ 